\title{
COMPUTATIONAL CHEMISTRY APPROACH TO SPACE CHEMISTRY
}

\author{
Y. ELLINGER \\ Ecole Normale Supérieure et Observatoire de Paris \\ 24 Rue Lhomond \\ 75005 Paris \\ France
}

\begin{abstract}
This review paper presents the results of state of the art Quantum Chemistry calculations in the field of Astrochemistry. It provides selected examples to illustrate the possible contribution of molecular orbital theories to solving a number of problems of astrophysical interest ranging from identification of new molecules to IR emission analysis and rate constants determinations.
\end{abstract}

Many of the roles played by laboratory work in the sequence going from the identification of a new molecule in space to the understanding of its chemistry, such as analysis of rotational spectra, evaluation of abundances, IR signature and possible mechanisms of formation and destruction, can be played by theoretical methods. Quantum Chemistry is ideally suited for this task as it refers directly to isolated gas phase systems and can be used to examine chemical species and processes that are difficult, if not impossible, to study in laboratories. Selected examples are presented below to illustrate how efficient a partner Quantum Chemistry can be for understanding chemistry in space.

\section{Identifying Molecular Structures: the $\mathrm{SiC}_{2}$ problem}

The astrophysical importance of $\mathrm{SiC}_{2}$ has been known for a long time. Though not identified, the electronic absorption spectrum was first observed in 1926 in the atmosphere of carbon stars. It was not until 1956 that laboratory experiments proved that the carrier of these bands was a molecule formed of $\mathrm{C}$ and $\mathrm{Si}$ atoms exclusively. On the basis of a partial vibrational analysis, the spectrum was assigned to $\mathrm{SiC}_{2}$; by analogy with a cometary spectrum of $\mathrm{C}_{3}, \mathrm{SiC}_{2}$ was assumed to be linear, and the absorption bands interpreted in terms of a ${ }^{1} \Pi \leftarrow{ }^{1} \Sigma^{+}$transition ${ }^{1}$. On the other side, theoretical arguments based on qualitative relations between the geometry of a molecule and the number of its valence electrons (Walsh's rules) pointed to a possibly non-linear structure.

This problem was definitively solved through a close collaboration between experiments and theory. The analysis of the visible spectra of jet cooled $\mathrm{SiC}_{2}$ showed ${ }^{2}$ that the rotational structure was inconsistent with a linear structure but, as demonstrated by ab-initio configuration interaction (CI) calculations ${ }^{3}$, came from a triangular structure, more stable by $1.1 \mathrm{kcal} / \mathrm{mol}$ than the linear. The correct assignement of the spectrum to an ${ }^{1} B_{2} \leftarrow{ }^{1} A_{1}$ was then possible. 
Experimental

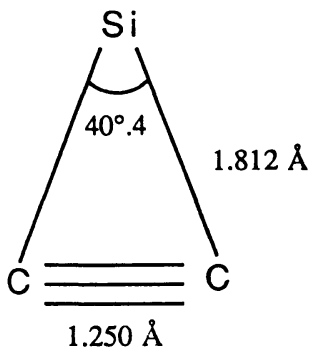

Ab-initio theory

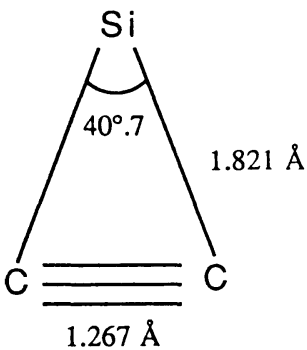

It should be emphasized that a single configuration description of this system gives the reversed stability order, namely, linear more stable than cyclic by $1.5 \mathrm{kcal} / \mathrm{mol}$, erroneously. Elaborate MCSCF calculations are necessary to provide orbitals of high enough quality to initiate the correlation energy calculations.

\section{Probing Intuitive Spectroscopic Models: the structure of $\mathrm{C}_{2} \mathrm{H}_{3}$}

One of the most important progenitors in the formation of hydrocarbons in ion-molecule reactions is protonated acetylene. The structure of this ion has been a puzzling problem for many years. Two geometries have been postulated on intuitive grounds, respectively called the linear and the bridge structures. Recently, high resolution infra-red studies have been possible ${ }^{4}$. Interpretation of the spectra using a simple internal rotor model in which the triangle formed by the three hydrogens rotates around the center of gravity of the $C=C$ bond leads to an "experimental" determination of the energy difference of $6.0 \mathrm{kcal} / \mathrm{mol}$ between the two structures.

Exptl. intuitive models

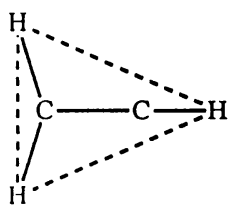

linear

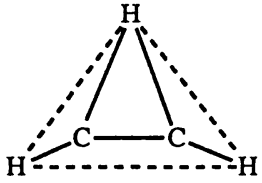

bridge
Ab-initio theory<smiles>C1CC1</smiles>

calculated

Ab-initio state of the art calculations 5 showed unambiguously that protonated acetylene was effectively more stable in a bridge structure, but with a geometry fundamentally different from that postulated in the spectroscopic model. The correct energy difference, i.e., $3.7 \pm 1.3 \mathrm{kcal} / \mathrm{mol}$ has been established. 


\section{Assessing Electronic States : the $2 \Sigma-2 \Pi$ alternative in linear radicals}

The discovery in IRC +10216 , by Guélin et $\mathrm{al}^{6}$. of a series of "doublets of roughly similar intensity, with center frequencies almost exactly in the ratio of half interger number and with doublet splitting regularly increasing with frequency" pointed, together with additional spectroscopic arguments and abondance considerations, to the conclusion that the carrier was a linear ${ }^{2} \Pi$ radical. Comparing the observed effective rotational constant of $1386.2 \mathrm{MHz}$ with an a-priori estimation using standard bond lengths suggested the $\mathrm{C}_{6} \mathrm{H}$ or $\mathrm{C}_{5} \mathrm{~N}$ radicals. However, if the parent closed-shell molecules have an obvious ${ }^{1} \Sigma$ ground state, radicals obtained by rupture of a terminal $\mathrm{CH}$ bond can be either $2 \Sigma$ or $2 \Pi$. The usual rule of thumb connecting the parity of the number of conjugated centers to the electronic state of the system ( $n$ odd $\rightarrow{ }^{2} \Pi$; $n$ even $\rightarrow{ }^{2} \Sigma$ ) which was verified for all the already known radicals predicts a $2 \Sigma$ state for both $\mathrm{C}_{6} \mathrm{H}$ or $\mathrm{C}_{5} \mathrm{~N}$ which cannot account for astronomical observations.

Exploratory calculations at the single configuration level of wave function showed a striking difference between the dipole moments of $2 \Sigma$ or ${ }^{2} \Pi$ radicals in the two series. A large value $(\mu=4.3 \mathrm{D})$ was found for the $2 \Pi$ of $\mathrm{C}_{6} \mathrm{H}$, a small one $(\mu=0.9 \mathrm{D})$ for the nearby ${ }^{2} \Sigma$ state; the opposite situation was found for $\mathrm{C}_{5} \mathrm{~N}$, i.e., a very small dipole $(\mu=0.07 \mathrm{D})$ for the ${ }^{2} \Pi$ state and a large one $(\mu=3.2 \mathrm{D})$ for the ${ }^{2} \Sigma$. As the line strengths scale with $\mu^{2}$, it was clear that the ${ }^{2} \Pi$ state could not be that of $\mathrm{C}_{5} \mathrm{~N}$. The attention was then focused on the $\mathrm{C}_{\mathrm{n}} \mathrm{H}$ series.

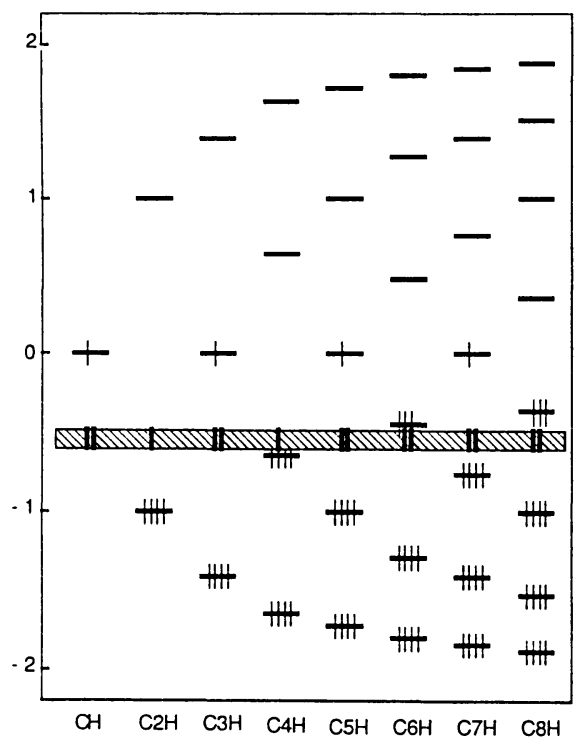

Molecular energy diagram for the $\pi$ system and terminal $\sigma$ orbital in the $C_{n} H$ series. The stripped area gives the expected position of the $\sigma$ orbital 
The energy diagram reported here shows how the energies of the $\pi$ orbitals are sensitive to the number of conjugated centers in the linear chain. By contrast, the $\sigma$ orbital pointing opposite to the carbon backbone is not affected by the length of the chain and its energy is constant. It is clear from this qualitative picture that all systems with an odd number of centers are undoubtedly $2 \Pi$. For even numbers of centers, the symmetry of the electronic ground state depends on the relative position of the upper $\pi$ shell with respect to the $\sigma$ orbital on the terminal carbon. The intuitive chemical view which looks at the molecule as a series of conjugated triple bonds gives the correct answer only up to 2 acetylenic fragments. The energy of the upper $\pi$ shell increasing regularly with the number of centers in the delocalized system, one can expect a ${ }^{2} \Sigma$ to ${ }^{2} \Pi$ crossing. A series of ab-initio large scale CI calculations designed to insure convergence in the basis set and in the n-particle space reached the conclusion that the state crossing occurs for 6 carbons with $2 \Pi$ more stable than $2 \Sigma$ by $0.25 \mathrm{eV}$; n-particle spaces up to $381566(2 \Sigma)$ and $375217(2 \Pi)$ configurations were considered in this study. Finally, it can be anticipated that all members of the $\mathrm{C}_{n} \mathrm{H}$ series with $\mathrm{n} \geq 6$ ( $n$ odd or even) have a ground state with 1 or 3

electron in the $\pi$ open shell according to the parity of the carbon chain 7 .

\section{Predicting Rotational Constants : the example of linear species}

Ab-initio molecular orbital theory can also be used to estimate the rotational constants for systems that are candidates for discovery in interstellar space. Since the precision needed for that purpose is well beyond the present capability of computational chemistry for medium size systems, evaluation of the rotational constants is made by combining ab-initio quantum chemistry calculations and observed data on the starting members of the series under investigation. The principle of these estimations is to derive an analytic or numerical fitting relation between the optimized theoretical geometries giving $\mathrm{Be}$ (theory) and the observed Bo. Such an approach has been used to predict rotational constants of the "next" members in the cyanopolyyne family ${ }^{8}$, the polycarbon monoxides ${ }^{8}$ and in the radical series ${ }^{9} \mathrm{C}_{\mathrm{n}} \mathrm{N}$ and $\mathrm{C}_{\mathrm{n}} \mathrm{H}$ :

$$
\begin{array}{cl}
\mathrm{B}_{0}\left(\mathrm{HC}_{13} \mathrm{~N}\right)=0.1073 \pm 0.0002 \mathrm{GHz} ; & \mathrm{B}_{0}\left(\mathrm{C}_{7} \mathrm{H}\right)=0.873 \pm 0.002 \mathrm{GHz} \\
\mathrm{B}_{0}\left(\mathrm{C}_{5} \mathrm{O}\right)=1.36 \pm 0.02 \mathrm{GHz} ; & \mathrm{B}_{0}\left(\mathrm{C}_{4} \mathrm{~N}\right)=2.428 \pm 0.003 \mathrm{GHz}
\end{array}
$$

with error bars tight enough to guide both laboratory and astronomical searches.

\section{Interpreting IR Bands: the PAH hypothesis}

The emission lines observed in many interstellar infrared sources at $3050,1610,1300$, 1150 and $885 \mathrm{~cm}^{-1}(3.3,6.2,7.7,8.6$, and 11.3 microns $)$ are hypothesized to originate from polycyclic aromatic hydrocarbon molecules (PAHs) 10,11 . These assignments are based on the analysis of laboratory infrared spectra of neutral PAHs. But, although the IR emission band spectrum resembles what one might expect from a mixture of PAHs, it does not match in details (frequency, band profile, or relative intensities) with the absorption spectra of any known PAH molecule.

Moreover, it is often assumed that PAHs, in the regions where they are observed, are mostly ionized, i.e., positively charged, and partly dehydrogenated ${ }^{12}$. If PAHs are considered as free flyers, the deductions made from the comparison of the interstellar emission with laboratory data are highly questionable. The spectra which are compared are 
spectra of different molecules: no a-priori reason can be quoted to get the same IR spectra, especially concerning intensities, for a molecule and its ionized or dehydrogenated derivatives. These systems which are generated from the parent PAHs are highly unstable in laboratory conditions. Experiments specifically designed for their study are extremely difficult to realize. Another source of information to test the PAH hypothesis is ab initio molecular orbital theory. It can be used to compute, from first principles, the geometries, vibrational frequencies, and vibrational intensities for model PAH compounds.

The IR spectra of naphtalene $\left(\mathrm{C}_{10} \mathrm{H}_{8}\right)$, anthracene $\left(\mathrm{C}_{14} \mathrm{H}_{10}\right)$, pyrene $\left(\mathrm{C}_{16} \mathrm{H}_{10}\right)$ and the corresponding positive ions have been calculated as models for linear and compact PAHs. These calculations showed that, if frequencies are little displaced, intensities by contrast are strongly affected by ionization; their variations are related to the type of vibration but similar in all the molecules calculated. The main reult is the decline of the ratio of the $\mathrm{CH}(3.3 \mu)$ to $\mathrm{CC}(7.7 \mu)$ intensities with ionization. The ratios to the other bands are in much better agreement with the observed ones in the cations while out of range for the neutral systems ${ }^{13}$. Dehydrogenation as been studied on the simplest PAH, naphtalene, and results are summarized in the following table.

Naphtalene : integrated intensities normalized to the $7.7 \mu$ band

\begin{tabular}{|c|c|c|c|c|c|}
\hline & $\mathrm{I}(3.3 \mu) / \mathrm{I}$ & $5.2 \mu) / \mathrm{I}(7.7 \mu)$ & $\mathrm{I}(6.2 \mu) / \mathrm{I}(7.7 \mu)$ & $\mathrm{I}(11.3 \mu) / \mathrm{I}(7.7 \mu)$ & $\mathrm{I}(11.3 \mu) / \mathrm{I}(7.7+8.6 \mu)$ \\
\hline $\mathrm{C}_{10} \mathrm{H}_{8}$ & 9.09 & & 2.82 & 15.27 & 8.00 \\
\hline $\mathrm{C}_{10} \mathrm{H}_{7}$ & 4.50 & & 1.56 & 10.37 & 6.83 \\
\hline & 5.42 & & 2.67 & 11.25 & 5.87 \\
\hline $\mathrm{C}_{10} \mathrm{H}_{6}$ & 2.30 & 0.04 & 1.70 & 5.81 & 3.75 \\
\hline & 2.00 & 0.11 & 0.81 & 4.63 & 3.0 \\
\hline $\mathrm{C}_{10} \mathrm{H}_{8}+$ & 0.02 & & 0.58 & 0.27 & 0.14 \\
\hline $\mathrm{C}_{10} \mathrm{H}_{7}+$ & 0.04 & & 0.49 & 0.34 & 0.18 \\
\hline & 0.04 & & 0.43 & 0.22 & 0.12 \\
\hline $\mathrm{C}_{10} \mathrm{H}_{6}+$ & 0.06 & 0.10 & 0.44 & 0.22 & 0.12 \\
\hline & 0.04 & 0.19 & 0.37 & 0.22 & 0.10 \\
\hline Observec & $0.084^{a}$ & $0.054^{\mathrm{a}}$ & $0.54^{a}$ & $0.29^{a}$ & $0.125^{b}$ \\
\hline
\end{tabular}

a) Ref. 14

b) Ref. 15

It is clear that the neutral PAH, dehydrogenated or not, cannot account for the observed intensity ratios, whereas the dehydrogenated positive ions compare favourably with the IR emission lines. The major result of this study 16 is that, if UIR bands originate from PAHs, then, these species are ionized and there is no longer the need for a massive dehydrogenation ${ }^{17}$ to accomodate the IR emission. 


\section{Checking Molecular Abundances: the $\mathrm{HCO}^{+} / \mathrm{CO}$ and $\mathrm{HCS}^{+} / \mathrm{CS}$ ratios}

Molecular abundances in interstellar clouds depend on the balance between a series of formation and destruction mechanisms whose relative importance cannot be easily established. Among those, dissociative recombination is an important process which is generally fast and governs the abundances of positive ions. The study of such a type of process, including the determination of associate reaction coefficients is an enormous task even at room temperature and the collision rates used in astrochemical models are low temperature extrapolated values obtained using more or less reliable procedures.

A typical challenge in this field was the difference of several orders of magnitude ${ }^{18,19}$ between the abundance ratios $\mathrm{HCO}^{+} / \mathrm{CO}$ and $\mathrm{HCS}^{+} / \mathrm{CS}$. The well admitted analogy between oxygen and sulphur chemistry being unable to give any rationalization of these facts, Millar suggested ${ }^{20}$ that the abundance ratio $\mathrm{HCS}^{+} / \mathrm{CS}$ could be higher, provided the dissociative recombination rate for $\mathrm{HCS}^{+}$was much lower than for $\mathrm{HCO}^{+}$.

The potential energy curves of the systems $\left(\mathrm{HCO}^{+}+e^{-}\right)$and $\left(\mathrm{HCS}^{+}+e^{-}\right)$in Rydberg and dissociative valence states, as well as those of the positive ions, were computed taking into account correlation effects in appropriate CI calculations ${ }^{21}$.
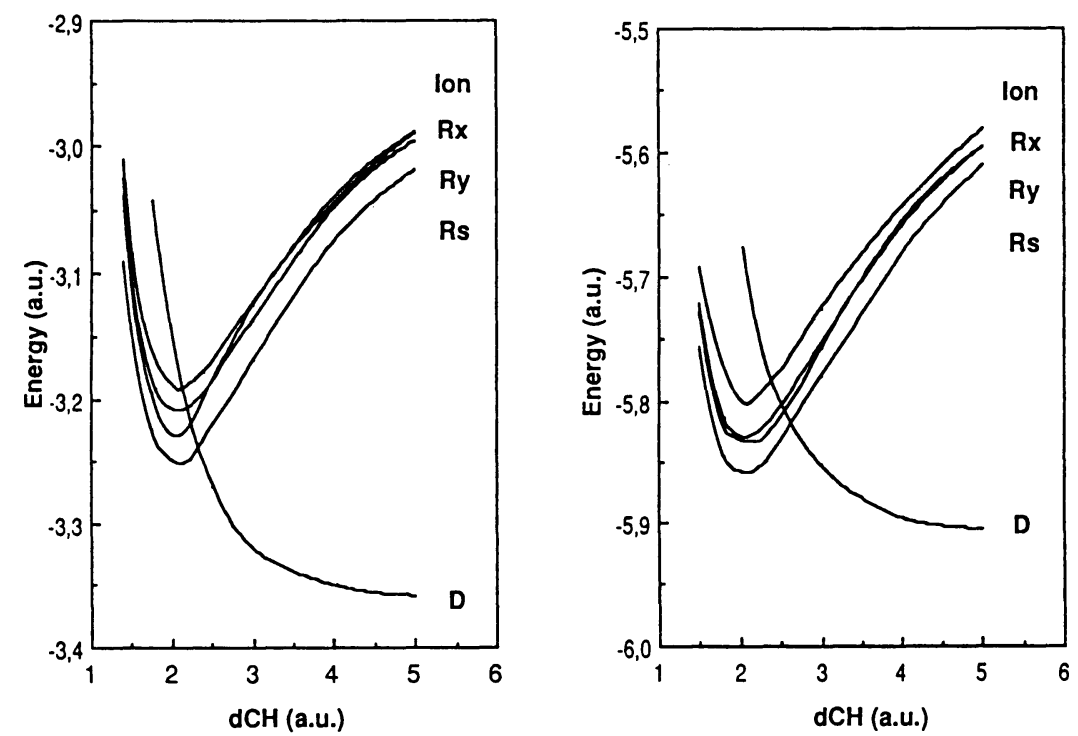

The quasi-diabatic representation of the potential surfaces shows striking differences:

- the crossing of the ground state surface of the ion with the dissociative curve of the neutral species is much closer to the energy minimum for HCO than for HCS.

- every term of the Rydberg series crosses the dissociative valence state at a position closer to the energy minimum for $\mathrm{HCO}$ than for $\mathrm{HCS}$.

Numerical calculations of the rate constants show that

- HCO has a usual behavior with a preponderant direct mechanism.

- HCS is anomalous, showing quite large contributions from an indirect mechanism.

The recombination rate of electrons with $\mathrm{HCO}^{+}$is significantly larger, by one to two orders of magnitude, than with $\mathrm{HCS}^{+}$. These $\mathrm{ab}$-initio calculations proved that Millar's hypothesis is perfectly justified. 


\section{Explaining Anomalous Reaction Rates : the $\mathbf{N H}_{3}++\mathrm{H}_{2}$ reaction}

Experiments show that the ion-molecule reaction

$$
\mathrm{NH}_{3}++\mathrm{H}_{2} \rightarrow \mathrm{NH}_{4}++\mathrm{H}
$$

is slow at room temperature, becomes slower as the temperature decreases, and then begins to increase in rate at still lower temperatures ${ }^{22,23}$. The authors hypothesized the mechanism for that reaction to be the initial formation of a complex, from which tunneling under a small transition state barrier could then occur. To understand the mechanism, both a quantum chemical study of the potential energy surface governing the reaction and a study of the reaction dynamics were undertaken ${ }^{24}$. The minimum energy pathway, taking vibrational energies into account, has been calculated using correlated wave functions and large basis sets.

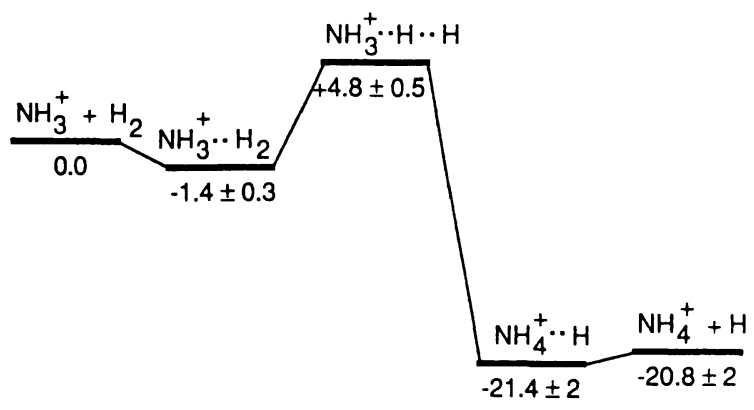

It proceeds through a weakly bound entrance channel complex $\mathrm{NH}_{3}+. . \mathrm{H}_{2}$, of binding energy $1.4 \pm 0.3 \mathrm{kcal} / \mathrm{mol}$, then rises to a transition state $\mathrm{NH}_{3}+$.H.H, of energy $4.8 \pm 0.5$ $\mathrm{kcal} / \mathrm{mol}$ above that of the reactants, before going down to the products via a weakly bound exit channel complex with an overall exothermicity of $25.6 \pm 0.7 \mathrm{kcal} / \mathrm{mol}$. This energy profile was then used in a theoretical treatment of the reaction dynamics by the phase space approach. The calculated rate coefficients are in quantitative agreement with experiment and reproduce the unusual temperature dependence observed in the laboratory.

\section{Concluding remarks}

The state of the art in Quantum Chemistry has now reached a point such that the wave functions determined by advanced computational techniques are accurate enough to provide reliable informations useful for a basic understanding of a number of chemical problems of astrophysical interest.Thus:

- Spectroscopic constants and rotational excitation of molecules that are difficult to study experimentally, can be calculated.

- Separations between spectroscopic states, as well as dipole moments necessary to determine column densities, can be obtained for exotic radicals.

- IR signatures can be calculated for neutral, ionized and dehydrogeenated PAHs, leading to a appealing modelisation of the UIR bands .

- Reaction mechanisms can be understood in greater detail than is generally possible from experiments alone; the role of tunneling can be analyzed easily. 
- Reaction rates to be used in chemical models can be evaluated directly from chemical dynamics calculations instead of being estimated by extrapolating room-temperature rates to interstellar temperatures.

Time has come for a fruitful collaboration between Astrophysics and Quantum Chemistry.

\section{References}

1. Gausset, L., Herzberg, G., Lagerqvist, A., and Rosen, A., (1965) Ap. J., 142, 45

2. Michalopoulos, D.L., Geisic, M.E., Landridge-Smith, P.R.R., and Smalley, R.E., (1984) J. Chem. Phys., 80, 3556

3. Grev, R.S., and Schaeffer III, H.F., (1984) J. Chem. Phys., 80, 3552

4. Crofton, M.W., Jagod, M.F., Rehfuss , B.D., and Oka, T., (1989) J. Chem. Phys., 91, 5139

5. Lindh, R., Rice, J.E., and Lee, T.J., J. Chem. Phys., (1991) 94, 8008

6. Guélin, M., Cernicharo, J., Kahane, C., Gomez-Gonzalez, J., and Wamsley, C.M., (1987) Astron. Astrophys., 175, L5

7. Pauzat, F., and Ellinger, Y., (1989) Astron. Astrophys., 216, 305

8. DeFrees, D.J., and McLean, A.D., (1989) Chem. Phys Lett., 158, 540

9. F. Pauzat, F., Y. Ellinger, Y., and McLean,A.D., (1991) Ap. J., 369, L13

10. Leger A. and Puget J.L., (1984) Astron. Astrophys., 137, L5

11. Allamandola L.J., Tielens A.G. and Baker J.R., (1985) Ap. J. Lett., 290, L25

12. Puget J.L. and Leger A., (1989) Ann. Rev. Astron. Astrophys., 27,161

13. DeFrees, D.J., Miller, M.D., Talbi, D., Pauzat, F., and Ellinger, Y.,Ap. J., 000

14. Cohen, M., Tielens, A.G., Bregman, J., Witteborrn, F.C., Rank, D.M., Allamendola, L.J., Wooden D.H., and de Muizon, M., (1989)Ap. J., 341, 246

15. Zavagno A., Cox P., and Baluteau J.P., (1992) Astron. Astrophys., 000

16. Ellinger, Y., Talbi, D., and Pauzat, F., (to be published)

17. de Muizon M., d'Hendecourt L.B. and Geballe T.R., (1990) Astron. Astrophys., 227, 526

18. Mitchell, G.F., Ginsburg, J.L., and Kuntz, P.J., (1978) Ap. J, Suppl., 38, 39

19. Thaddeus, P., Guélin, M., and Linke, R.A., (1981) Ap. J. Lett., 246, L41

20. Millar, T.J., Adams, N.G., Smith, D., and Clary, D.C., (1985) M.N.R.A.S., 216, 1025

21. Talbi, D., Hickman, A.P., Pauzat, F., Ellinger, Y., and Berthier, G., (1989) Ap. J., 339, 231

22. Luine, J.A., and Dunn, G.H., (1985), Ap. J. Letters, 299, L67

23. Bohringer, H., (1989) Chem. Phys Lett., 122, 185

24. Herbst, E., DeFrees, D.J., Talbi, D., Pauzat, F., Koch, W., and McLean, A.D., (1991) J. Chem. Phys., 94, 7842 